\title{
A Distributed Algorithm for Maximizing Linear Tree Density for One to Many Wireless Communication
}

\author{
James Pope \\ Department of Computer Science \\ C4I Center \\ George Mason University \\ 4400 University Dr, Fairfax, VA 22030 \\ james.h.pope8@gmail.com
}

\author{
Robert Simon \\ Department of Computer Science \\ C4I Center \\ George Mason University \\ 4400 University Dr, Fairfax, VA 22030 \\ simon@gmu.edu
}

\begin{abstract}
Reliable and efficient broadcast functions are essential in large, dense, multi-hop low power wireless systems managed by a gateway. Previous approaches addressing this issue rely on centralized and/or solutions requiring complex implementations. This paper describes the Deal algorithm for minimizing transmissions and hop count while maintaining reliability in a distributed fashion. The algorithm is compatible with and easily integrated into current low power, lossy network protocols.

We first present Deal as a centralized algorithm and evaluate using numerical simulations. We then derive a distributed algorithm appropriate for low power, lossy networks. We evaluate the distributed algorithm using a testbed with over 300 nodes. Our results demonstrate that Deal is reliable and energy efficient. The experiments show that Deal significantly reduces the required number of transmissions for network broadcast in large low power, lossy networks.
\end{abstract}

\section{CCS Concepts}

-Networks $\rightarrow$ Network protocol design; Network reliability; Ad hoc networks; Routing protocols;

\section{Keywords}

Low Power, Lossy Networks, Wireless Broadcast, Internet of Things

\section{INTRODUCTION}

The Internet of Things (IoT) technologies are currently experiencing significant growth [20]. Specifically, low power, lossy networks (LLNs) enable large groups of devices to connect with the internet [14]. Large LLNs typically have a coordinator (a.k.a. base station, sink) that manages communication in and out of the LLN. The size of the networks necessitate the need to communicate over multiple hops re-

Permission to make digital or hard copies of all or part of this work for personal or classroom use is granted without fee provided that copies are not made or distributed for profit or commercial advantage and that copies bear this notice and the full citation on the first page. Copyrights for components of this work owned by others than ACM must be honored. Abstracting with credit is permitted. To copy otherwise, or republish, to post on servers or to redistribute to lists, requires prior specific permission and/or a fee. Request permissions from permissions@ acm.org.

PE-WASUN'16, November 13-17, 2016, Malta, Malta

(C) 2016 ACM. ISBN 978-1-4503-4505-7/16/11 . \$ $\$ 15.00$

DOI: http://dx.doi.org/10.1145/2989293.2989296 quiring higher layer protocol support. LLNs are dominated by two communication patterns, one to many and many to one. The many to one pattern addresses devices needing to communicate information (e.g. sensor readings) to the sink and has been largely solved by gradient routing protocols [21], [19]. Reliable and efficient one to many communication has yet to be sufficiently addressed, particularly for larger LLNs. Potential future applications will inevitably require the need to communicate beyond the range of a sink and require larger networks than are currently supported today. The fact that large LLNs are not common is likely due to lack of support from current protocols and approaches, motivating this research.

An obvious goal is for the communication to be reliable. Specific for one to many communication, this means that when a source sends a message all the intended recipients receive the message. In our context, reliability is measured by the packet delivery rate (PDR) and it is not necessary that every message be delivered.

Two additional goals of one to many LLN communication are to minimize the number of transmissions and minimize the latency. We previously defined the linear tree density metric $\left(\Lambda_{m}\right)$ to simultaneously measure these two goals [15]. The previous approach to achieve these goals used Kruskallike algorithms that maximized $\Lambda_{m}$. Kruskal's algorithm generates a spanning tree by connecting a forest of trees. Prim's algorithm generates a spanning tree by starting with one vertex in a tree and adding an edge at each step growing the tree until it connects all reachable vertices [17].

This paper focuses on a Prim-like approach, the Deal algorithm, that creates spanning trees and shows how this could be implemented in a distributed fashion using RPL, the current standard for IPv6 LLNs. The primary contributions of this paper are Prim-like approaches to creating spanning trees that maximize $\Lambda_{m}$, a distributed variant compatible with RPL, and practical results implementing the approach with a test bed.

We evaluated our approach using numerical simulations. A distributed variant of Deal was then derived and implemented using an open source implementation of the RPL protocol. The distributed algorithm was evaluated using a test bed with three different topologies with sizes from 62 341 nodes. The numerical and noteworthy test bed results confirm the efficacy of the approach.

The paper first presents some background and related work followed by the system model. The centralized Deal algorithms are next presented along with numerical results. 
The distributed Deal algorithm is then presented with implementation details. Finally, the distributed algorithm is evaluated using a test bed.

\section{BACKGROUND AND RELATED WORK}

Our work centers on tree-based wireless networks using low power communication architectures such as those supported by the IEEE 802.15.4 specification [10]. These systems are populated by resource constrained devices, have unreliable communication links and low data rates, and are referred to as low-power lossy networks (LLNs) [14]. These networks are actively managed by a sink or gateway. Systemwide broadcasting initiated by the sink is critical for the operation of LLNs. Competing goals of reliable delivery versus excessive transmission remain a challenge for LLNs.

There have been several proposals for LLN broadcasting [12]. One important class of protocols is exemplified by the Trickle approach, which uses a gossiping and re-transmission strategy [13]. Similar to work in this paper, the HASTE algorithm [15] addresses the linear tree density problem using a Kruskal-like algorithm. Flooding protocols, such as Net flood [5], have also been proposed for reliable, energy efficient network broadcasts though typically require each node to transmit at least once. La, et al. [11], evaluate various approaches for addressing the problem using multicast relay mechanisms attempting to reduce retransmissions for constrained networks.

Coding techniques have also been proposed to address one to many broadcasting. Rahnavard, et al., [16] propose a rateless coding approach to provide reliability and energy efficiency in multi-hop wireless networks.

Tang and Wang [18] proposed a broadcast tree to address reliable, energy efficient one to many communication for security purposes. Leveraging the existing spanning tree created by RPL has been explored by Duquennoy, et al. [6]. The authors show how the RPL spanning tree can be used to support arbitrary communication patterns.

\section{SYSTEM MODEL}

The system model is based on wireless, ad-hoc networks with static (or slowly changing) topologies. The model assumes an existing many to one communication pattern that is typically used for data traffic from the nodes towards the sink. Correspondingly, there is the need to disseminate information using one to many communication typically from the sink to the nodes in the network.

Acknowledgment messages are typically used by unicast transmissions to achieve reliability. However, a fundamental assertion is that broadcast transmissions using explicit acknowledgments for reliability are not appropriate for LLNs. This would mean that each device would send an acknowledgment message back to the sender (presumably with some contention avoidance mechanism). Even for sparse networks this scheme is very inefficient and could add significant congestion.

The model assumes a radio with an ideal, omni-directional transmission/reception range (interference is not considered). The following parameters are given.

\section{1. $V$ is the set of vertices in the graph $G$}

2. $\operatorname{sink} \in V$ vertex that originates broadcast messages
3. $E$ is the set of edges in the graph $G$

4. $T$ is a spanning tree derived from graph $G$

The desired objective is to minimize the number of transmissions necessary to broadcast a message to all the vertices in the graph. Given that leaf vertices do not transmit and only listen/receive the broadcast message, the objective is equivalent to maximizing the number of leaves in some spanning tree $T$ of $G$. Therefore, finding a spanning tree $T$ with the maximum number of leaves, known as a maximal leaf spanning tree (MLST), can result in fewer transmissions to reach all the vertices in the graph. The MLST is known to be NP-complete [8]. Later we show that this approach extends to reducing the number of transmissions to reach all of the nodes in the LLN. Generally, when referring to graphs we use the term vertex and when referring to a network we use the term node.

The linear tree density, denoted $\Lambda_{m}$, is defined in [15]. For clarity, it is briefly reiterated here. The linear tree density is the ratio of the number of leaves of a spanning tree to the maximum hop count in the spanning tree. Maximizing $\Lambda_{m}$ is equivalent to maximizing the number of leaves and minimizing the maximum hop count. The indicator variable $y_{v}$ is defined to be 1 if vertex $v$ is a leaf, 0 otherwise. The formulation increases as maximum hop count decreases and the number of leaves increases, capturing the desired combined objective.

\section{Maximize Linear Tree Density}

$$
\max \frac{\sum_{v=1}^{|V|} y_{v}}{\max \left\{\text { hopCount }_{\text {sink }}(v) \mid \forall v \in V\right\}}
$$

\section{DEAL ALGORITHM FOR LINEAR TREE DENSITY}

The previous HASTE approach, while effective, is centralized and would be difficult to derive as a distributed algorithm. Motivated by the need for an algorithm more convenient to being distributed, two Prim-like algorithms are proposed, denoted Deal $A$ and Deal B. Prim's algorithm starts with a single vertex in the tree and then grows the tree. This is very similar to how gradient routing protocols generate spanning trees. Though still centralized, we show later how Deal B could be distributed using current standard protocols. The approaches are shown in Algorithms 1 and 2 .

\subsection{Deal A Variant}

Deal A selects the parent that has the minimum path cost plus link cost. The algorithm is essentially Dijkstra's algorithm run on an undirected graph that has been transformed into a weighted directed graph. The link cost (i.e. edge weight) is calculated on line 19 by considering the ratio of the vertex's hop count and degree. The vertex then updates its own path cost to be its selected parent's path cost plus the link cost, accumulating to subsequent children. The hop counts for each vertex are determined by running a breadth first search at line 7 . A similar construction could have first transformed the input graph $G$ into a weighted directed graph $G^{\prime}$ and then simply run Dijkstra's algorithm 


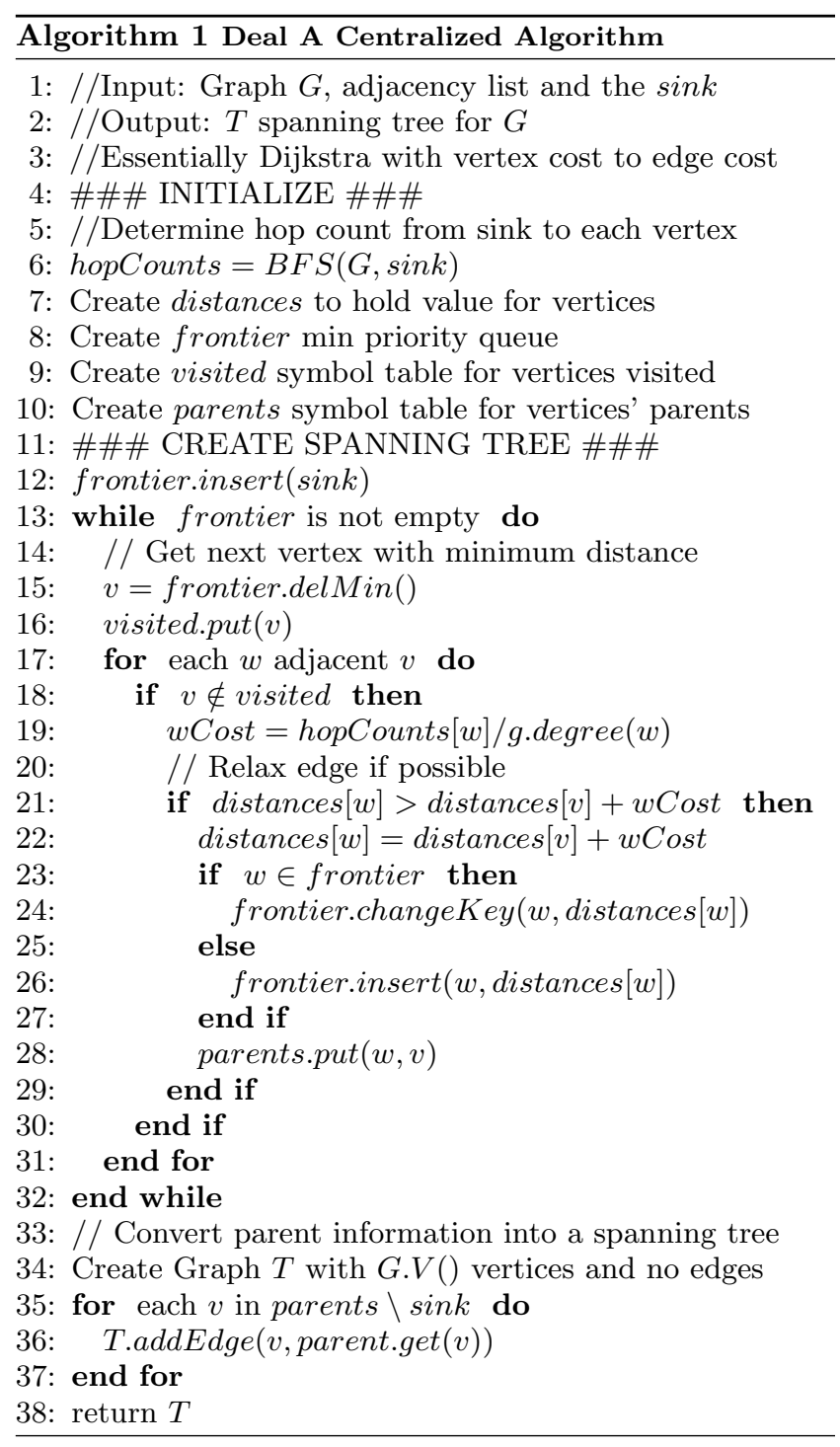

on $G^{\prime}$. The algorithm needs to convert the spanning tree implicitly defined in the parent's symbol table into a tree $T$. This is done at line 34 and $T$ is returned. A consequence of this approach is that a path with high degree vertices may be chosen over a path with fewer vertices of lower degree. Thus, it may select a path that is longer (i.e. non-optimal in terms of hop count) in an attempt to maximize leaves instead of minimizing hop count.

\subsection{Deal B Variant}

Deal B uses the hop count as the path cost and selects the parent with the highest degree. The node then updates its path cost to subsequent children as simply its parent's hop count plus one. This approach first minimizes the maximum hop count. By construction, it selects a spanning tree whose maximum hop count is the eccentricity of the graph. It then adjusts the spanning tree, without increasing any vertex's hop count, to maximize the number of leaves.

The shortest hop count spanning tree to the sink is first determined at line 5 . The for loop at line 10 partitions the vertices into disjoint sets based on their hop count. Note that this also orders the sets from closest to the sink to those

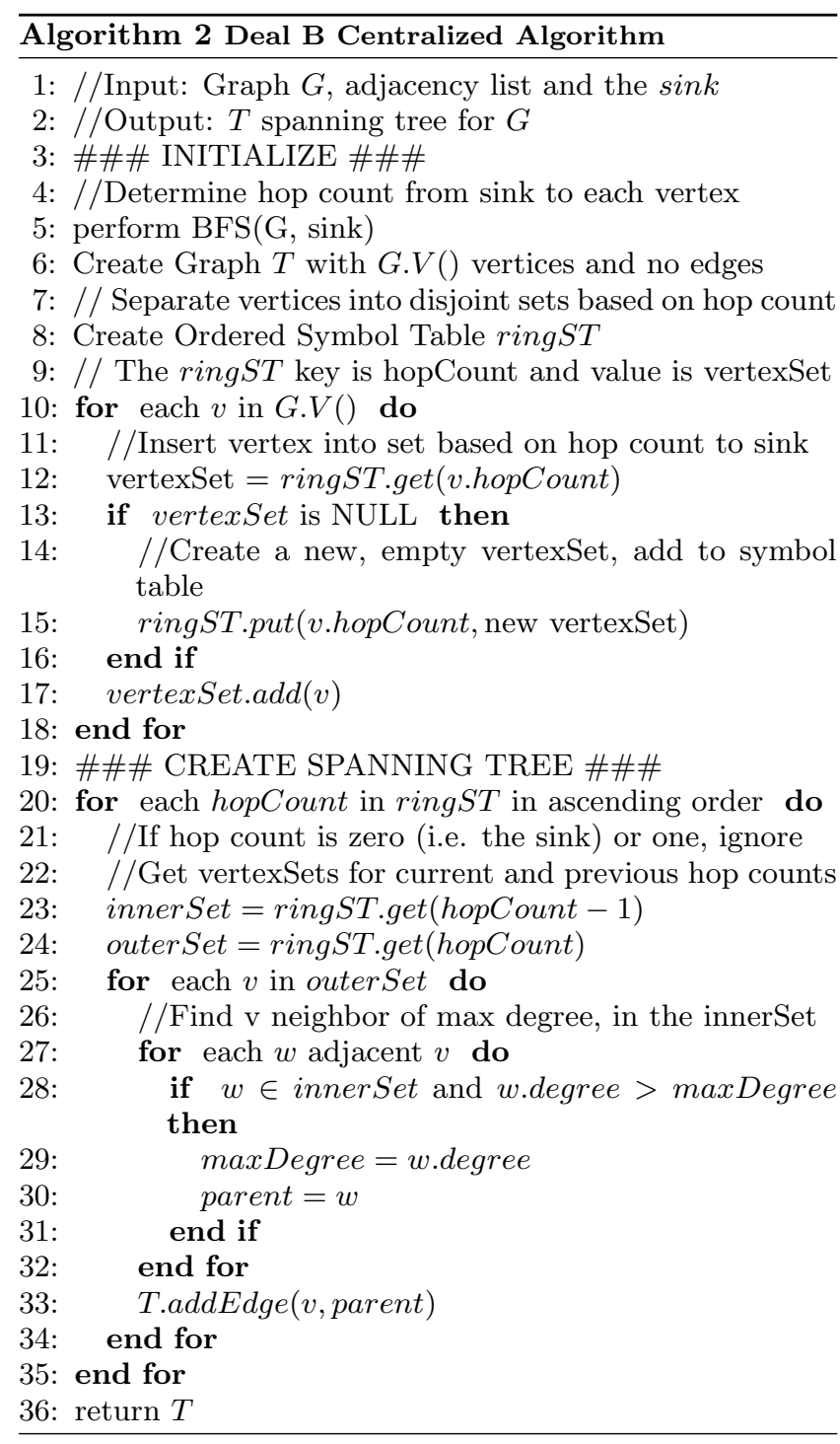

farthest away. The for loop at line 20 starts the creation of the spanning tree. Each iteration looks at vertices closer to the sink (innerSet) and their potential children one more hop from the sink (outerSet). The for loop at line 25 looks at each child in the outer set and finds the parent in the innerSet of highest degree. At line 33 the parent of the child is set in the output tree $T$. The parent can happen to be the same as the original shortest hop count spanning tree parent.

\subsection{Time Complexity Analysis}

We consider the time complexity analysis for both variants using worse case. Throughout the analysis we consider the time complexity for symbol table operations to be a constant and priority queue insert/delMin operations to be logarithmic [17].

Deal A's while loop at line 13 creates the spanning tree and is similar to Dijkstra's Eager algorithm with the exception of the calculation of the link cost at line 19, that can be taken as a constant. Thus creating the spanning tree requires $E \times l g(V)$ [17]. During initialization, line 7 requires $V+E$ with the remaining initializations requiring $V$ or less. 
Creating the final tree $T$ at line 34 requires $V$. Thus, Deal A's overall time complexity for the initialization, spanning tree creation, and conversion is $O([V+E]+[E \times l g(V)]+$ $[V]) \equiv O(E \times \lg (V)+E+V)$.

Deal B's initialization at line 5 similarly requires $V+$ $E$. Considering a linear topology (worse case), partitioning/sorting the vertices into hop count sets at line 10 requires $V \times \lg (V)$. The for loop at line 19 creates the spanning tree. Instead of counting loops, which would be overly pessimistic, we examine the maximum number of times a vertex and edge can be examined. Each vertex is examined exactly one time at line 25. Each edge is examined at most once at line 27. Edges connecting vertices to other vertices in the same hop count set are not examined. Thus creating the spanning tree requires $V+E$, comporting with our intuition that Deal B is a second pass over the BFS spanning tree that adjusts the selected parent. Deal B's overall complexity is $O([V+E]+[V \times \lg (V)]+[V+E]) \equiv O(V \times \lg (V)+E+V)$

Table 1: Deal Centralized Algorithm Analysis

\begin{tabular}{cc}
\hline & Time Complexity \\
\hline Deal A & $E \times \lg (V)+E+V$ \\
Deal B & $V \times l g(V)+E+V$ \\
\hline
\end{tabular}

The summary of the worse case analysis for both variants is shown in Table 1.

\section{NUMERICAL SIMULATION}

A series of tests were run using two different graph densities, defined by increasing the range of each vertex. The first range setting of 150 results in a sparser graph with an average of 6.3 neighbors with a 1000 vertex graph. The second range setting of 300 results in a denser graph with an average of 25 neighbors for 1000 vertices. Each graph is produced using a random seed. We take the number of samples to be $n=100$ and use $99 \%$ confidence intervals. The results are based on the sink location being a random, uniformly selected vertex. The Deal variants were compared to HASTE and BFS generated spanning trees along with the upper bound (denoted UB).

The upper bound can be determined by considering the maximum number of leaves and the eccentricity of the graph. The maximum possible number of leaves can be determined by $|V|-($ eccentricity -1$)$. The shortest maximum hop count possible for any spanning tree is the eccentricity of the graph. The eccentricity is determined by taking a breath first search on the graph and then taking the maximum hop count. Thus an upper bound on the linear tree density metric is $\frac{|V|-(\text { eccentricity }-1)}{\text { eccentricity }}$.

BFS was chosen for naive comparisons. We expect the results to be between BFS and UB, ideally closer to UB. Figures 1 and 2 show the $\Lambda_{m}$ results for each algorithm for network sizes from 100-2000 in increments of 100 .

Figure 1 shows that HASTE performs slightly better than Deal A and Deal B, though within the margin of error, and all perform better than BFS. There is a considerable difference between Deal B and UB suggesting potential for improvement. The optimal value has to be between the Deal $\mathrm{B}$ and UB. Though the difference between Deal B and UB is considerable, without further analysis, it is unknown how much further improvement can be achieved.

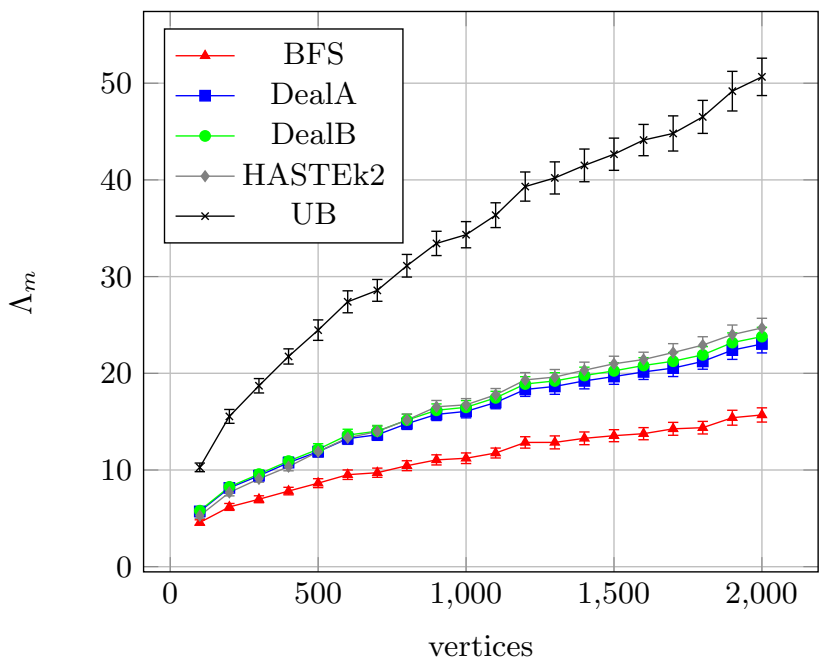

Figure 1: Sparse $\Lambda$ Simulation Results

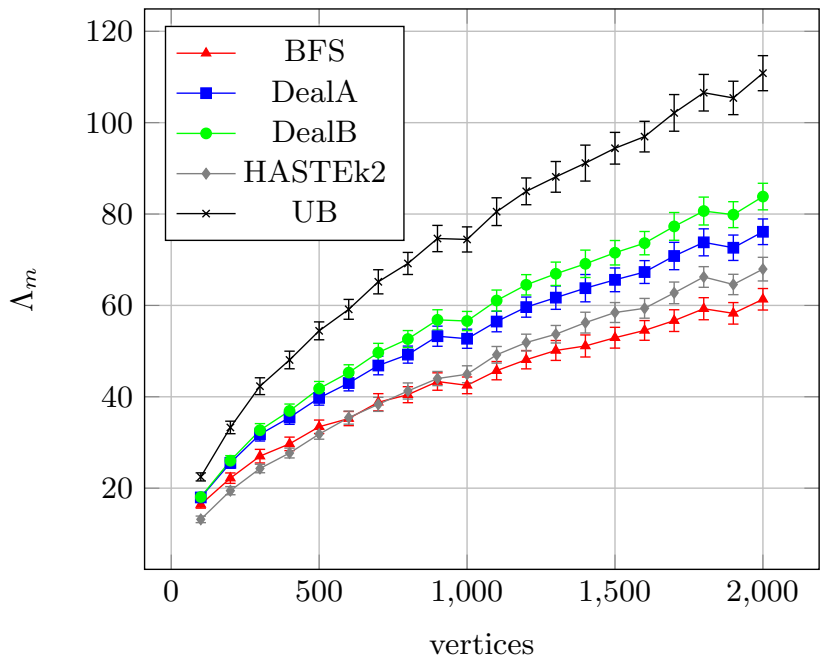

Figure 2: Dense $\Lambda$ Simulation Results

Figure 2 shows that Deal B clearly outperforms Deal A and HASTE for larger networks. Furthermore, it is within $70 \%$ of the optimal at 2000 vertices. The $\Lambda_{m}$ metric does not explicitly weight the preference of leaves versus maximum hop count. However, reducing the hop count by one has a more significant effect than increasing the leaf count by one. The difference between Deal A and B is believed to be the result of $\Lambda_{m}$ favoring a smaller maximum hop count, noting that Deal B's maximum hop count is less than or equal to Deal A's maximum hop count.

For sparse networks, the Deal algorithms do not appear to be any more effective than HASTE. This could be simply be due to few decisions available for parent selection. For more dense networks, the Deal algorithms perform well even for smaller networks.

\section{DISTRIBUTED DEAL ALGORITHM}

Though the previous algorithms are centralized, with some modification, they are amenable to being distributed. This section describes how Deal B could be distributed. Deal B 
was modified to be compatible with gradient, many to one routing protocols such as RPL [19], CTP [9], and RIME [5]. The Distributed Deal Algorithm is designed to address three issues. The first issue is to determine neighboring vertices and the link quality from them. The second issue is to allow a node to determine if it is a leaf and should retransmit network broadcasts. The third issue is to create a spanning tree by each node selecting a parent that minimizes the cost to the sink.

The first issue is addressed by neighbor advertisement messages that assist in maintaining a neighbor table including link quality. The neighbor table contains the neighbor's address, degree, parent address, ewma_ed, and occupied fields for each neighbor. All fields except for occupied are 16 bit unsigned values. The occupied value is an 8 bit unsigned value with 0 representing unoccupied and 1 meaning occupied. The addresses used are the lower 2 bytes of the assigned IPv6 address (for the test bed these are guaranteed to be unique). Note that the approach could have been further integrated with the RPL neighbor table by adding the degree, parent, and ewma_ed fields, potentially saving memory.

For the second issue, when a network broadcast is received by a node it must determine whether or not it is a leaf. Given each neighbor's parent from the neighbor table, the node is a leaf if it is not the parent of any of its neighbors.

The third issue is solved by using RPL. RPL generates a spanning tree using DIO messages [19] and allows parent selection using an objective function. The potential parents are maintained by RPL and presented to the objective function for comparisons. The algorithm was integrated into the Contiki Operating System's RPL implementation [4].

The Distributed Deal Algorithm is broken into three related processes and an objective function. The three processes are the neighbor advertisement transmit, neighbor advertisement receive, and network broadcast receive.

\subsection{Neighbor Advertisement Transmit}

The neighbor advertisement process is shown in Algorithm 3. It starts before the other processes (called from the Contiki main process before the event loop), initializes the neighbor table, and schedules a transmit at a random time between $[0,16000]$ milliseconds. When the timer expires, a neighbor advertisement message is transmitted, the transmit window is doubled, and another transmission is scheduled (e.g. random time in [0,32000] milliseconds). This continues indefinitely but the doubling stops at the MAX_ADV_WINDOW of 16 minutes. The neighbor table is sized to be the same as the RPL neighbor table set by MAX_NEIGHBORS. To handle very dense networks this was set to 128 . The neighbor advertisement messages contain the nodes address, number of neighbors (i.e. the degree of the node), and parent address ( 0 if no parent yet from RPL).

Thus, each node learns the link quality from its neighbor to itself. Links are not necessarily symmetric and the network broadcasts need quality links from a node to its neighbors. To avoid a more complicated exchange of information, this approach assumes the links are symmetric.

\subsection{Neighbor Advertisement Receive}

The neighbor advertisements serve to provide each node with the degree, parent, and link quality of each of its neigh-
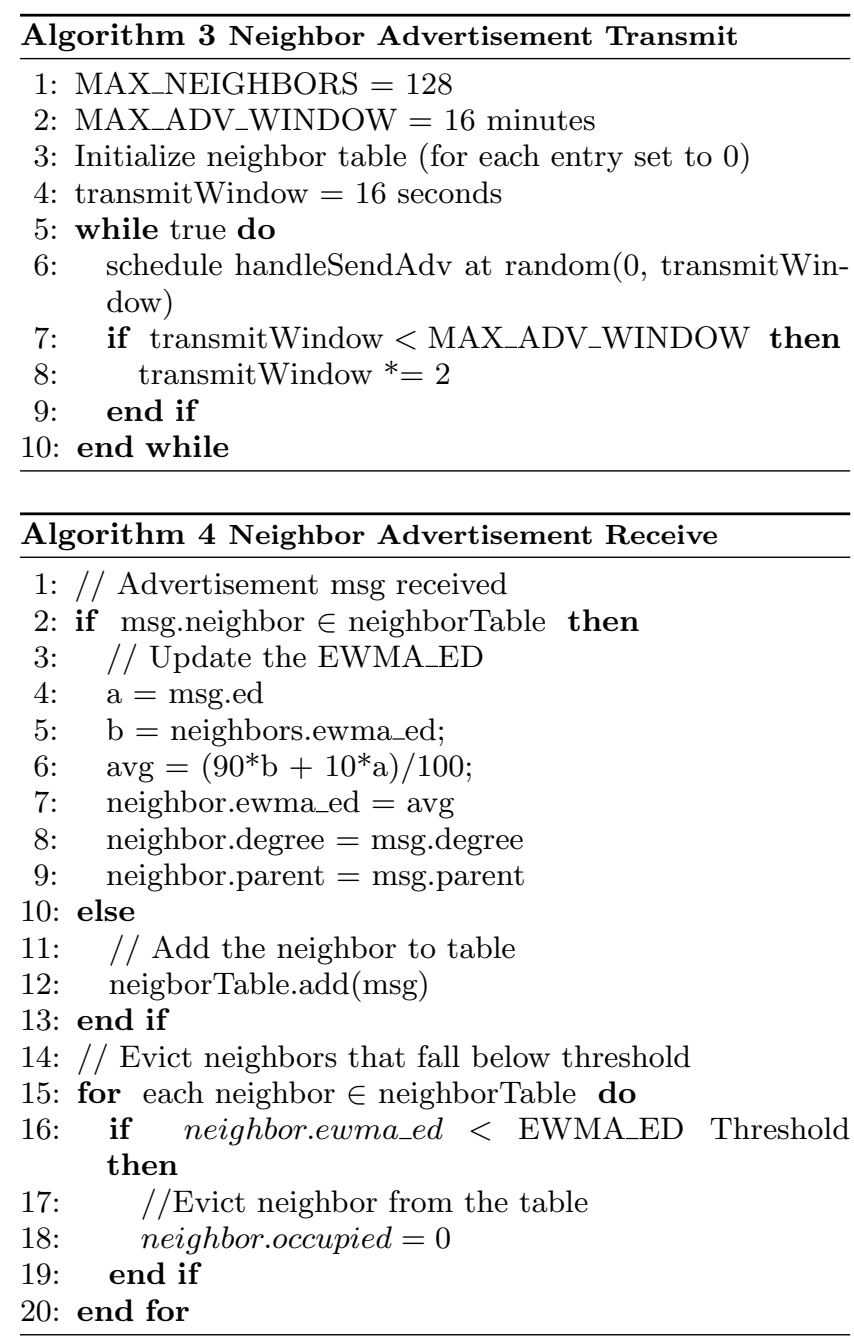

bors. It is important to have a measure of the quality of the link between a node and its neighbors. We use the energy detected (ED) measurement provided by the RF231 radio [2] to provide an indication of the link quality for each received neighbor advertisement message. The RF231 radio driver was updated to record the ED, a value in $[0,84]$, when receiving the transmission. The ED measurement is calculated by averaging the received signal strength (RSSI) of the last eight symbols for a single message. To account for ED measurements across multiple messages, an exponentially weighted moving average (EWMA-ED) is calculated with $\alpha=0.1$. The updated ED measurement for a neighbor is calculated as:

$$
\text { ewma_ed }=((1-\alpha) \times \text { oldED })+(\alpha \times \text { newED })
$$

Subsequent neighbor advertisements from the same neighbor result in updating the neighbor's averaged ED measurement, degree and parent.

The Neighbor Advertisement Receive process is shown in Algorithm 4. At line 2, when a network advertisement message is received the node checks to see if it already knows the neighbor. Lines 4 to 7 show the ED value being updated using $\alpha$. The code assumes no floating point so values are multiplied by 100 incurring some minor error. If the neigh- 
bor is new and there is room in the neighbor table, it is simply added to the neighbor table at line 12 . To ensure the link to neighbors remains good, a threshold is checked each time the neighbor table is updated at line 16 . If the EWMA_ED falls below the threshold, the neighbor is evicted from the neighbor table at line 18 .

\subsection{Network Broadcast Receive}

The network broadcast receive process is shown in Algorithm 5 . The sink will initiate a network broadcast by transmitting the message to its neighbors. The network broadcast message will include a sequence number (abbreviated seqno) for each new network broadcast. Shown at line 4, when a network broadcast is received the node checks its neighbor table to determine if any neighbors have it selected as their parent. If so, this means the node is the parent of some other node and therefore not a leaf. If the node is a leaf then the message is passed to the application layer and processing ceases.

Non-leaf nodes check the broadcast message to ensure it is a new broadcast by examining the seqno at line 9 . If the seqno is newer, then the number of transmissions is reset to zero, the current buffer is set to the new broadcast, and at line 14 an initial send is randomly scheduled between [0, 1000] milliseconds. Though not shown, when the send function is called it will transmit the message and double the transmitWindow and again randomly schedule another send within the transmitWindow. It does this until the transmitWindow exceeds the MAX_SEND_WINDOW. Based on the settings shown, each non-leaf node will retransmit the network broadcast message at least once and no more than three times.

If the seqno is the same as the current seqno and the source of the message is not the original source of the message, then we assume another neighbor has received and transmitted the message. This acts as a passive acknowledgment and the node cancels the current send at line 20 . If the seqno is older then it is ignored.

\subsection{Deal RPL Objective Function}

Ideally the Distributed Deal Algorithm would closely match and converge with the Deal B Centralized variant. This would require a mechanism to present all nodes closer to the sink along with their degree so that a node could select the parent with the highest degree. RPL is designed to minimize the cost to the sink and, using the objective function, will prefer parents with a lower path cost + link cost, with some heuristics to minimize route flapping. The Contiki OS RPL plug-in objective function architecture does not allow for this more direct implementation. In Contiki OS, the RPL objective functions are set up to compare each neighbor against other neighbors one at a time.

To avoid modifying the architecture, the Deal objective function was implemented to compare the two presented neighbors in a similar fashion as Deal B. The function is designed to select parents from the neighbor table that have a high degree and a low hop count. It is based on the ofo objective function (i.e. minimizing hop count) with a modification to select the neighbor closer to the sink and with the highest degree. The Deal objective function compares two neighbors using the following cost computation, where the minimum rank increase (MRI) is 256 as defined by RPL:
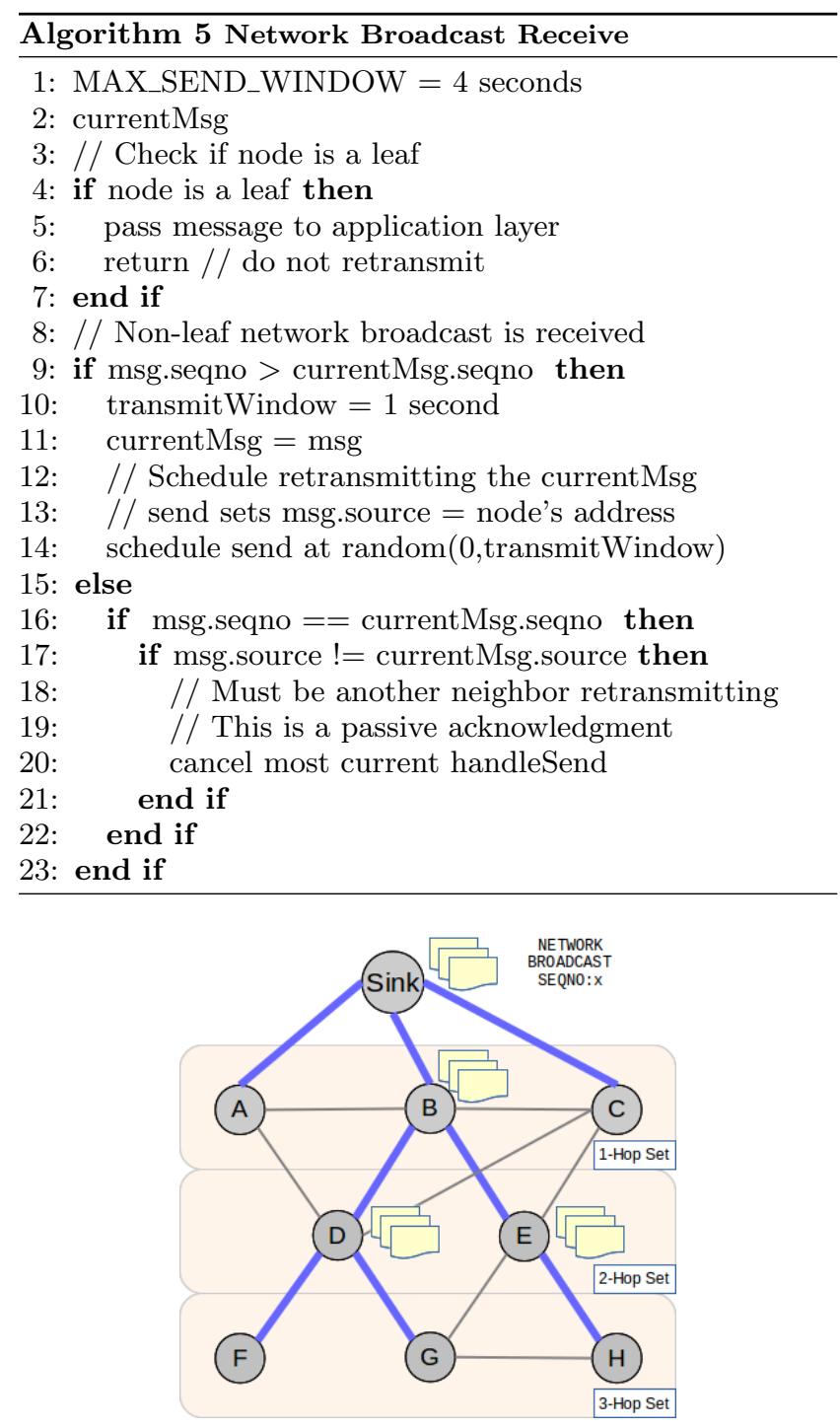

Figure 3: OF-Deal Spanning Tree

$$
v_{\text {cost }}=\left(v_{H C} * M R I\right)+\left(M R I / v_{\text {degree }}\right)
$$

If the node has no neighbors then $v_{\text {degree }}$ is set to one. As the protocol expands from the sink, the hop count increases with each node rebroadcasting the additional hop, noting that the degree is not propagated in the path cost to other nodes. This formulation will prefer nodes that are one hop closer and with a higher degree.

Figure 3 depicts an example graph, the Deal induced spanning tree, and a subsequent network broadcast. Nodes D, E and $\mathrm{G}$ select parents one hop closer to the sink and with the highest degree. When a network broadcast occurs, only the sink and non-leaf nodes, B, D, and E, retransmit the message. The figure depicts each non-leaf node retransmitting up to three times. In this example, it takes between $[4,12]$ transmissions for each network broadcast.

\section{TEST BED EXPERIMENTS}

The approach was implemented using the FIT/IoT-LAB 


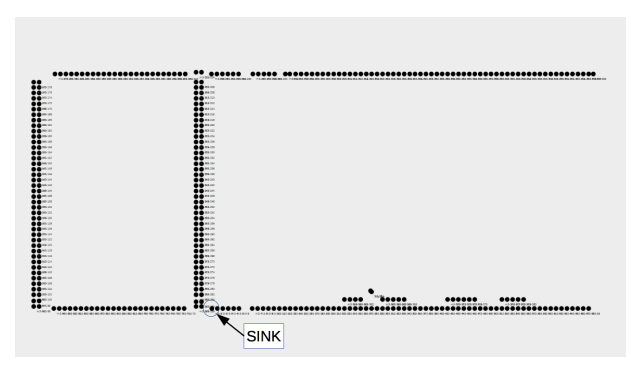

Figure 4: Topology Grenoble Test Bed

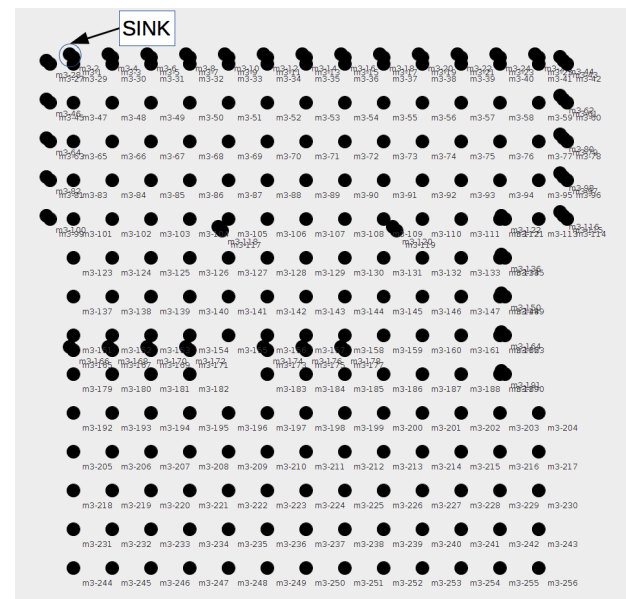

Figure 5: Topology Lille Test Bed

test bed [3]. The IoT-LAB provides a very large scale infrastructure facility suitable for testing small wireless sensor devices and heterogeneous communicating objects. Specifically, the M3 hardware configuration was used and consists of an ARM processor along with an Atmel RF231 $2.4 \mathrm{GHz}$ IEEE802.15.4 compliant radio [1].

\subsection{Test Bed Configuration}

Three test bed sites were used for the experiments with their topologies shown in Figures 4, 5, and 6. Each site has nodes on vertical poles so each position in this two dimensional representation may represent multiple nodes. Lille and Strasbourg are generally grid like topologies and care had to be taken to generate networks with multiple hops. The Grenoble topology is nearly linear and spread out more than the others resulting in a challenging use case.

Using the default $0 \mathrm{dBm}$ radio transmit power results in a star network for the Strasbourg site and nearly a star network for the Lille site. To create multi-hop networks, the neighbor density of the nodes had to be reduced. The neighbor density was affected by the radio transmit power, radio receive threshold, and the neighbor table EWMA_ED threshold. Table 2 shows the settings for each of the test bed sites along with the resulting average neighbor density. Note that the average density is similar to the numerical graphs considered in Section 5.

Each experiment consisted of a subset of the nodes in the the site. Table 3 shows the number of nodes, excluding the sink, used in the experiments for each site and the duration of the experiment. To achieve $100 \%$ delivery of a network broadcast, we expect to receive the number shown. For the

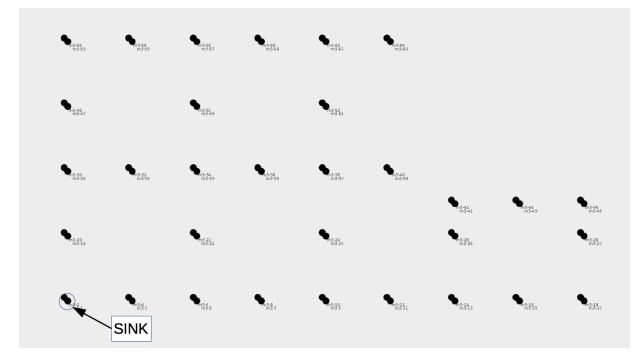

Figure 6: Topology Strasbourg Test Bed

Table 2: Test Bed Site Configuration

\begin{tabular}{ccccc}
\hline Test Bed & Transmit & Receive & \multicolumn{2}{c}{ EWMA_ED Average } \\
Site & Power & Threshold & Threshold & Density \\
\hline Grenoble & $0 \mathrm{dBm}$ & $-90 \mathrm{dBm}$ & 15 & 18 \\
Lille & $-17 \mathrm{dBm}$ & $-90 \mathrm{dBm}$ & 20 & 65 \\
Strasbourg & $-12 \mathrm{dBm}$ & $-90 \mathrm{dBm}$ & 22 & 25 \\
\hline
\end{tabular}

Lille and Strasbourg sites, the sink was chosen to be in a corner of the topology. For Grenoble the sink was chosen to be near the middle, lower line of Figure 4. All three experiments were run for one hour. Every minute the sink sends a network broadcast with an updated sequence number.

Table 3: Test Bed Experiment Configuration

\begin{tabular}{ccc}
\hline Test Bed & \# Nodes & $\begin{array}{c}\text { Duration } \\
(\min )\end{array}$ \\
\hline Grenoble & 340 & 60 \\
Lille & 234 & 60 \\
Strasbourg & 61 & 60 \\
\hline
\end{tabular}

\subsection{Network Stack}

The implementation was based on the example RPL many to one collection in the Contiki OS. The network stack was configured as follows.

1. Radio $=$ RF231

2. Radio Duty Cycling $=$ contikimac_driver

3. Media Access Control = csma_driver

4. Network Sublayer $=$ sicslowpan_driver

5. Network Layer $=$ ipv6

6. Transport Layer $=\mathrm{udp}$

Severe congestion issues were first encountered on the Lille and Strasbourg sites. By examining the log files, it was discovered that the csma_driver was frequently dropping packets due to its buffer being full. Only unicast messages requiring acknowledgment would occupy a buffer for a significant amount of time. Deal messages are broadcast and no other applications were configured. It was determined that the RPL DAO messages were causing the overflow. DAO messages are unicast to parents to notify them of downward routes for point to point communication.

In RPL storing mode (which is how Contiki implements DAO messages), each intermediate node must maintain information to know how to route to descendants. The DAO 


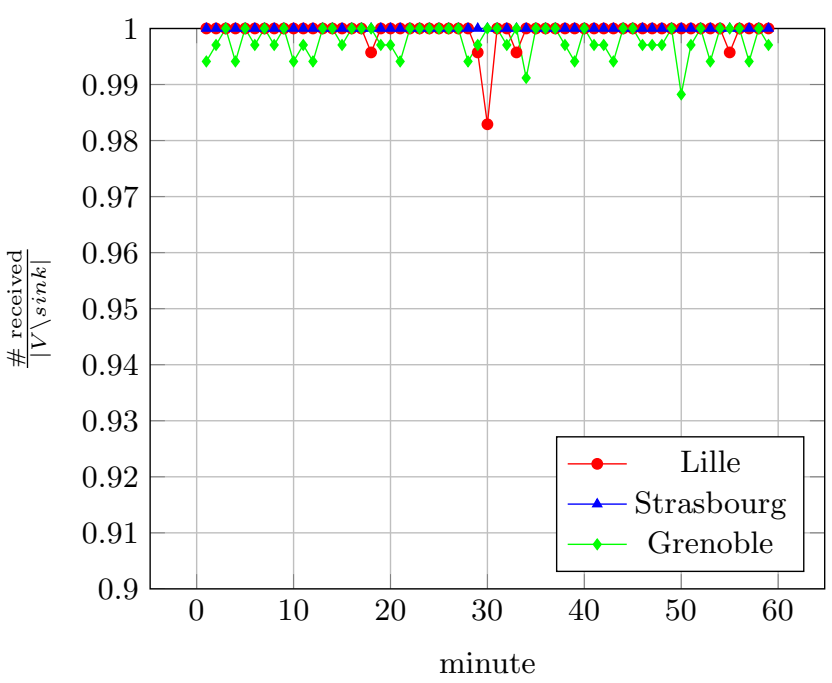

Figure 7: PDR

messages were large enough to require fragmentation. Note that this only occurred during large tests (e.g. over 100 nodes). It is believed that the DAO messages were being frequently generated in an attempt to maintain large subtrees resulting in the observed congestion collapse. For these experiments, the RPL DAO messages were removed as this communication pattern was not necessary. However, it suggests additional research for this mechanism.

\subsection{Reliability (PDR) Results}

Figure 7 shows the packet delivery rate for each broadcast for each experiment. It clearly shows the reliability achieved using this controlled flooding approach. The Lille experiment appears to experience some loss at the 30th broadcast and 33rd broadcast. Interestingly, this also shows in the latency results in Figure 8.

\subsection{Maximum Latency Results}

The test bed writes the print outs of each device to a log file along with a timestamp. The timestamp is the time the log was written and is totally ordered. This introduces some latency regarding when the log is written. The latency is calculated by taking that time the broadcast was logged by the receiver minus the time the broadcast was sent by the sink. This means that some error in logging latency is introduced but provides a global clock to compare the events.

The maximum latency results are shown in Figure 8. The figure shows the maximum latency for the Grenoble site to be higher than the other sites. This is expected considering the size, density and topology of the Grenoble site. The Strasbourg site has a lower density than Lille, however, is much smaller than the other sites and, therefore, experiences the least latency. Though it is unclear what caused the disruption, two broadcasts for the Lille site experienced significant latency.

\subsection{Efficient Network Broadcast Results}

The test bed can monitor the voltage, current, and power for each device. Unfortunately, the monitoring caused a scheduling issue with the test bed resulting in a deadlock

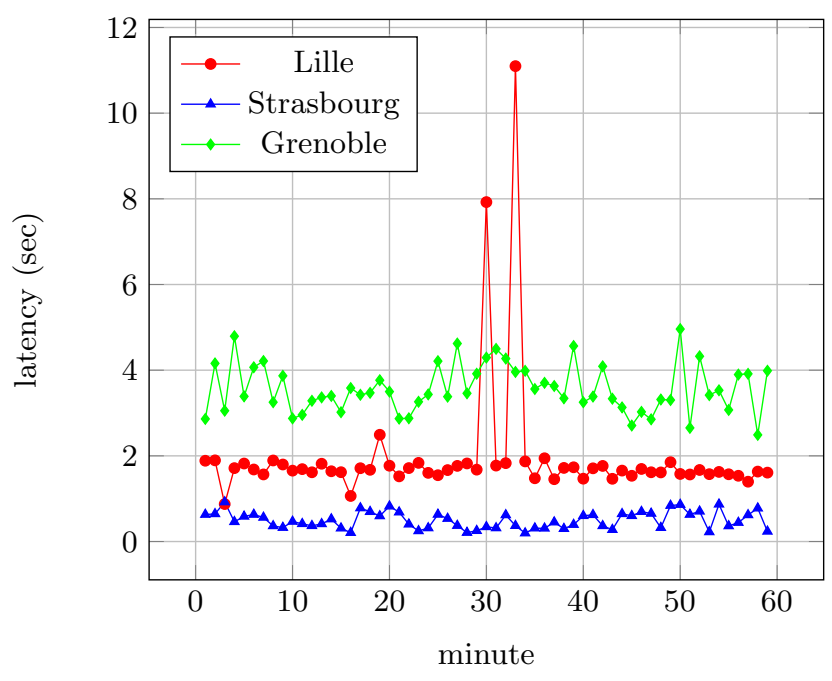

Figure 8: Maximum Latency

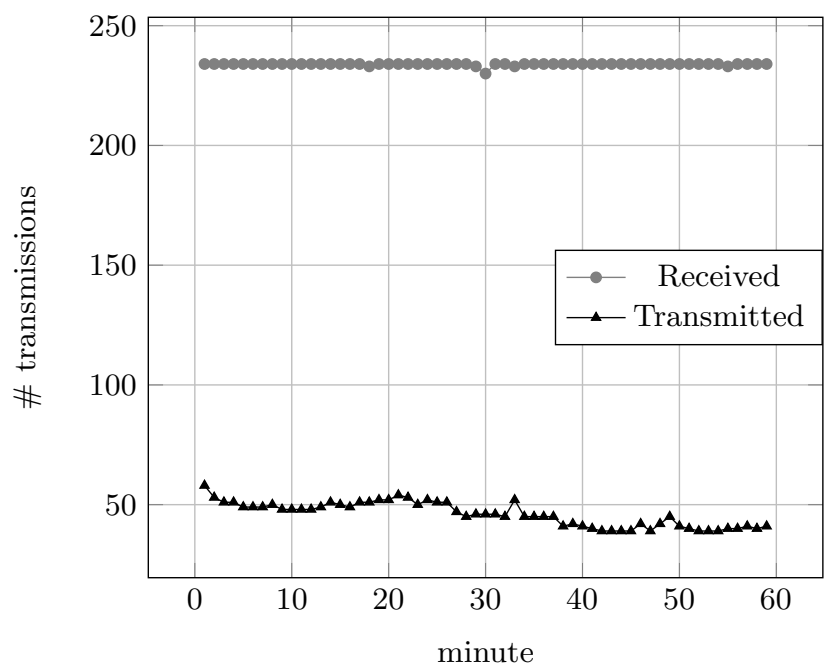

Figure 9: Lille Broadcast Results

for all experiments. In lieu of the power measurements, to demonstrate the efficiency of network broadcasts, the number of transmissions and the number of nodes receiving the network broadcast are plotted together for each broadcast sequence number. This is used as an indication of the energy necessary to broadcast a message. Figures 9, 10, and 11, show these for each site.

\subsection{Overhead}

The neighbor advertisement messages create overhead that decreases with time. Note again that this could be integrated non obtrusively into RPL DIO messages. The number of neighbor advertisement messages are determined by the process described in Algorithm 3. The number of messages exponentially decreases over time until a minimum is reached. The overhead for each experiment is shown in Figure 12. The results match our expectation and demonstrate that the overhead quickly becomes negligible. 


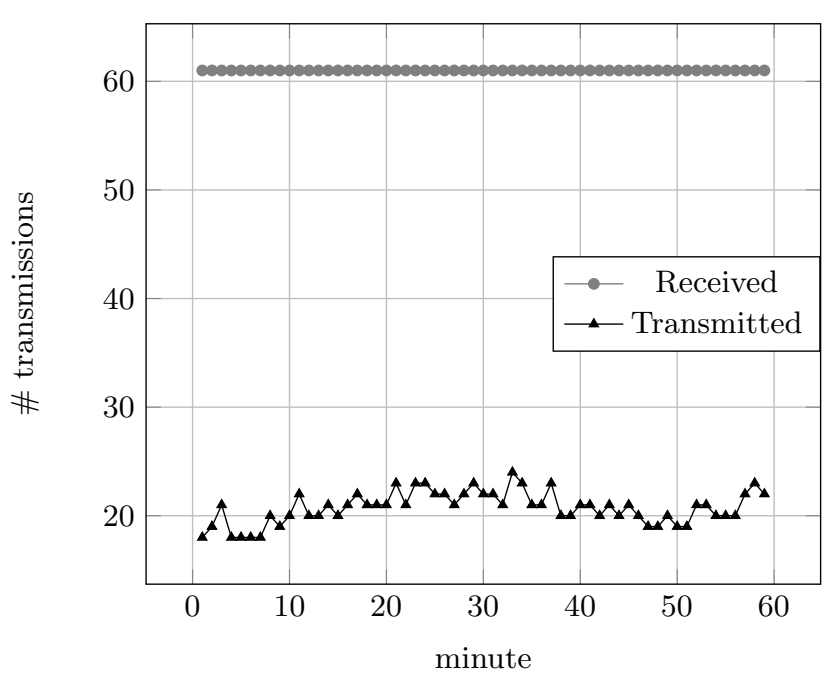

Figure 10: Strasbourg Broadcast Results

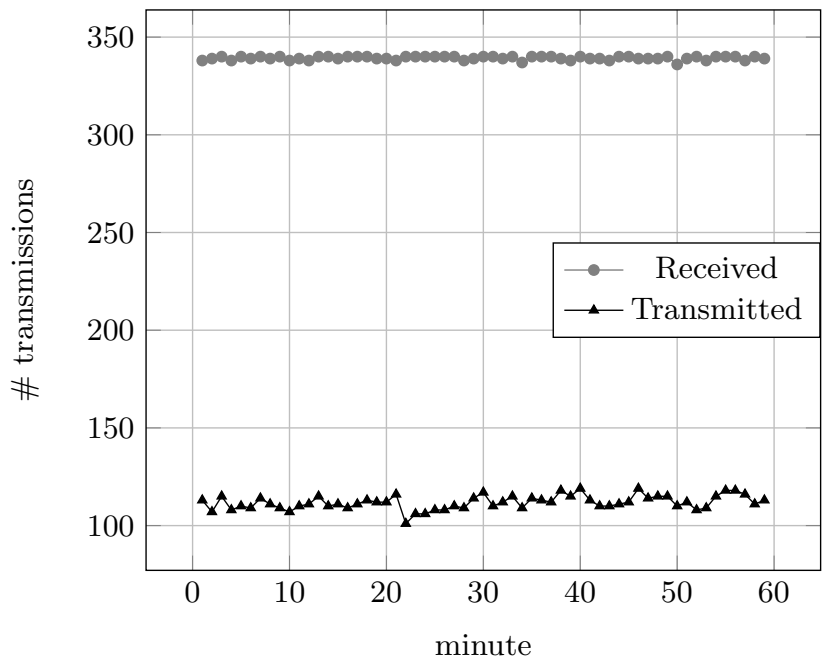

Figure 11: Grenoble Broadcast Results

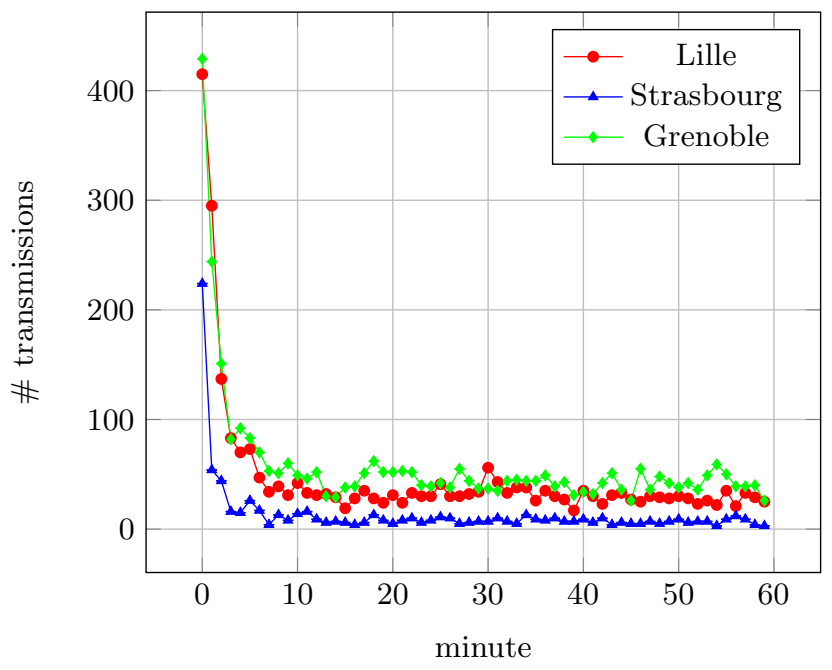

Figure 12: Deal Neighbor Overhead

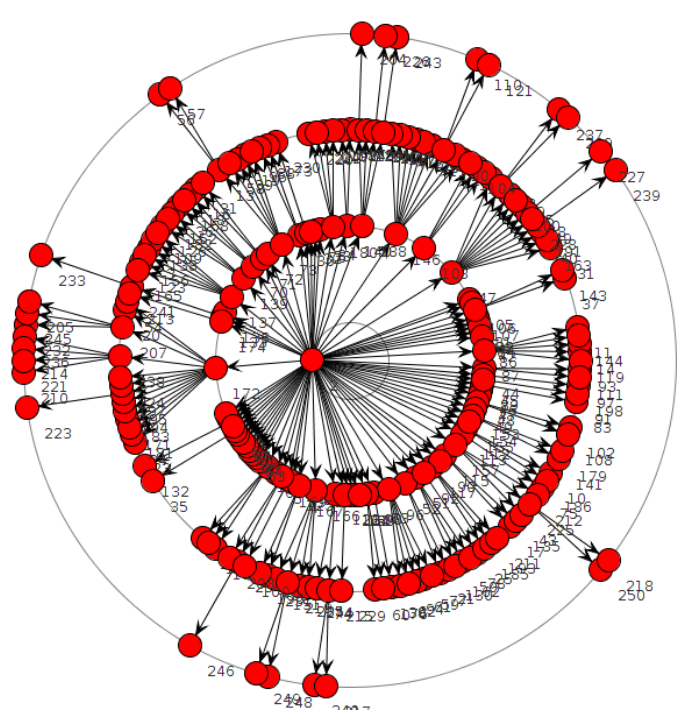

Figure 13: Lille Radial Spanning Tree View

\subsection{Spanning Tree Visualizations}

For analysis purposes, it was instructive to see the spanning trees using a graph visualization tool. We used the Java Universal Network/Graph (JUNG) [7] library to analyze the last reported spanning tree in the log files. The radial visualization from the sink outwards was the most helpful. Though visualizing over the physical topology was also useful, it was not as informative. Figure 13 shows the radial spanning tree for the Lille experiment.

\subsection{Future Work}

The neighbor advertisement messages operate in a similar manner to RPL DIO messages. Future work would be to integrate the selected parent and degree information into DIO messages (e.g. using the RPL metric container mechanism) in order to eliminate the neighbor advertisement overhead. Future work would also include comparing Deal against other flooding protocols and RPL objective functions.

The Deal approach suggests using two spanning trees, one for many to one and another for one to many communication. Exploring a single spanning tree appropriate for both communication patterns could also be considered.

As expected, it was found that the neighbor link quality measurement had a significant impact on the PDR. By using a higher threshold, better PDR results were obtained. The values used for the link quality threshold were found empirically for each site. However, restricting the neighbor links considered by using a higher threshold changes the graph. Though good PDR results, this more restricted graph can result in a poor linear tree density (i.e. higher hop count, more non-leaves) and more transmissions. More analysis is required to better understand the tradeoffs between reliability and linear tree density, perhaps by using a dynamic link quality threshold.

\section{CONCLUSIONS}

This paper examined the problem of one to many communication in large low power, lossy networks. The problem is very important for emerging IoT applications and difficult 
to solve. We designed a distributed algorithm from an effective centralized algorithm that maximized the linear tree density metric. The distributed algorithm was integrated with the current LLN IPv6 standard protocol. It was then implemented and evaluated on a large test bed with between 62-341 devices with impressive results. The approach is reliable and energy efficient in terms of radio transmissions, appropriate for LLN one to many communication.

\section{ACKNOWLEDGMENTS}

This work is supported by NSF under grants CNS-1116122 and CNS-1205453. Thanks to the FIT/IoT Lab administrators and users that assisted in debugging experiments. Additional thanks to Dana Richards, Noha Hazzazi and Arda Gumusalan for advice and feedback.

\section{REFERENCES}

[1] Iot-lab: a very large scale open testbed, 2016.

[2] Atmel. AT86RF231 Datasheet, 9 2009. Rev. C.

[3] C. Burin des Roziers, G. Chelius, T. Ducrocq, et al. NETWORKING 2011: 10th International IFIP TC 6 Networking Conference, Valencia, Spain, May 9-13, 2011, Proceedings, Part I, chapter Using SensLAB as a First Class Scientific Tool for Large Scale Wireless Sensor Network Experiments, pages 147-159. Springer Berlin Heidelberg, Berlin, Heidelberg, 2011.

[4] A. Dunkels, B. Gronvall, and T. Voigt. Contiki - a lightweight and flexible operating system for tiny networked sensors. In Proceedings of the 29th Annual IEEE International Conference on Local Computer Networks, LCN '04, pages 455-462, Washington, DC, USA, 2004. IEEE Computer Society.

[5] A. Dunkels, F. Österlind, and Z. He. An adaptive communication architecture for wireless sensor networks. In Proceedings of the 5th International Conference on Embedded Networked Sensor Systems, SenSys '07, pages 335-349, New York, NY, USA, 2007. ACM.

[6] S. Duquennoy, O. Landsiedel, and T. Voigt. Let the tree bloom: Scalable opportunistic routing with orpl. In Proceedings of the 11th ACM Conference on Embedded Networked Sensor Systems, SenSys '13, pages 2:1-2:14, New York, NY, USA, 2013. ACM.

[7] D. Fisher, J. O'Madadhain, and S. White. Jung: The java universal network/graph framework.

[8] M. R. Garey and D. S. Johnson. Computers and Intractability; A Guide to the Theory of NP-Completeness. W. H. Freeman \& Co., New York, NY, USA, 1990.

[9] O. Gnawali, R. Fonseca, K. Jamieson, D. Moss, and P. Levis. Collection tree protocol. In Proceedings of the 7th ACM Conference on Embedded Networked Sensor Systems, SenSys '09, pages 1-14, New York, NY, USA, 2009. ACM.

[10] I. IEEE. Part 15.4: Wireless medium access control (mac) and physical layer (phy) specifications for low-rate wireless personal area networks (wpans), 2011.

[11] C.-A. La, L.-O. Varga, M. Heusse, and A. Duda. Energy-efficient multi-hop broadcasting in low power and lossy networks. In Proceedings of the 17th ACM
International Conference on Modeling, Analysis and Simulation of Wireless and Mobile Systems, MSWiM '14, pages 41-50, New York, NY, USA, 2014. ACM.

[12] P. Levis, E. Brewer, D. Culler, D. Gay, S. Madden, N. Patel, J. Polastre, S. Shenker, R. Szewczyk, and A. Woo. The emergence of a networking primitive in wireless sensor networks. Communications of the ACM, 51(7):99-106, 2008.

[13] P. Levis, T. Clausen, J. Hui, O. Gnawali, and J. Ko. The Trickle Algorithm. RFC 6206 (Proposed Standard), Mar. 2011.

[14] P. Levis, A. Tavakoli, and S. Dawson-Haggerty. Overview of existing routing protocols for low power and lossy networks. IETF Draft Report, 2009.

[15] J. Pope and R. Simon. Efficient one-to-many broadcasting for resource-constrained wireless networks. In 2015 IEEE 40th Conference on Local Computer Networks ( $L C N$ ), Clearwater, USA, Oct. 2015.

[16] N. Rahnavard, B. N. Vellambi, and F. Fekri. Fts: A fractional transmission scheme for efficient broadcasting via rateless coding in multihop wireless networks. In MILCOM 2007 - IEEE Military Communications Conference, pages 1-7, Oct 2007.

[17] R. Sedgewick and K. Wayne. Algorithms, 4th Edition. Addison-Wesley, 2011.

[18] C. Tang and N. Wang. A lightweight energy-efficient reliable broadcast tree for wireless sensor networks. In Computer Research and Development (ICCRD), 2011 3rd International Conference on, volume 1, pages 373-378, 2011.

[19] P. Thubert. RPL: IPv6 Routing Protocol for Low-Power and Lossy Networks. RFC 6550 (Proposed Standard), Mar. 2012.

[20] J. Tully. Gartner says 6.4 billion connected "things" will be in use in 2016, up 30 percent from 2015. Technical report, Gartner, Inc., November 2015.

[21] T. Watteyne, A. Molinaro, M. G. Richichi, and M. Dohler. From manet to ietf roll standardization: A paradigm shift in wsn routing protocols. IEEE Communications Surveys Tutorials, 13(4):688-707, Fourth 2011. 